Research Paper

\title{
Gonadotropin-releasing hormone agonists, anti-androgens and the risk of cardio-cerebrovascular disease in prostate cancer patients: an asian population-based observational study
}

\author{
Jong-Mi Seong1, Dongho Shin², Jae Woo Sung ${ }^{2}$, Shinjay Cho², Jonghyup Yang ${ }^{2}$, Sungmin Kang 3 , Hyong
} Woo Moon², Kyu Won Lee², and U-Syn Ha ${ }^{2}$

1. Ewha womans university, Department of pharmacy, Seoul, Republic of Korea

2. Department of Urology, Seoul St. Mary's Hospital, College of Medicine, The Catholic University of Korea, Seoul, Republic of Korea

3. Department of Urology, Catholic Kwandong University College of Medicine, International St. Mary's Hospital, Incheon, Republic of Korea.

$\square$ Corresponding author: U-Syn Ha, MD, PhD. Department of Urology, Seoul St. Mary's Hospital, College of Medicine, The Catholic University of Korea, 222, Banpo-daero, Seocho-gu, Seoul, 06591, Republic of Korea. Tel: +82-2-2258-1401, Fax: +82-2-2258-1536 , E-mail: ushamd@catholic.ac.kr

(c) The author(s). This is an open access article distributed under the terms of the Creative Commons Attribution License (https://creativecommons.org/licenses/by/4.0/). See http://ivyspring.com/terms for full terms and conditions.

Received: 2019.07.07; Accepted: 2020.02.20; Published: 2020.04.06

\begin{abstract}
Purpose: To conduct a population-based study to determine whether the use of $\mathrm{GnRH}$ agonist and antiandrogens are associated with an increased risk of cardio-cerebrovascular disease (CCVD) in Asian patients with prostate cancer using the National Health Insurance Service-Elderly Cohort Database (NHIS-ECD).

Materials and Methods: We included a total of 2,413 men aged 60 years or older with prostate cancer between January 2003 and December 2008. Outcomes of interest included the first occurrence of cardiovascular events [acute myocardial infarction (AMI), ischemic heart disease (IHD)] and cerebrovascular events [ischemic stroke (IS), and cerebrovascular disease (CVD)].

Results: The 5-year AMI-free rates of patients diagnosed with prostate cancer and treated with $\mathrm{GnRH}$ agonists, antiandrogens alone, or androgen deprivation therapy (ADT)-naïve interventions were $97.0 \%, 96.5 \%$, and $98.3 \%$, respectively, while the 5 -year IHD-free rates were $93.2 \%, 92.3 \%$, and $94.5 \%$, respectively. Exposure to $\mathrm{GnRH}$ agonists or antiandrogen regimens did not significantly increase the risk of AMI or IHD compared to ADT-naive treatment in multivariate Cox proportional-hazards models after adjusting for other covariates. Five-year IS-free rates of patients exposed to $\mathrm{GnRH}$ agonists, antiandrogens alone, and those with ADT-naïve prostate cancer were 94.8\%, 94.7\%, and 95.5\%, respectively, while the five-year CVD-free rates were $92.9 \%, 93.3 \%$, and $94.6 \%$, respectively. Cox proportional-hazards models also failed to show that men who received $\mathrm{GnRH}$ agonist or antiandrogen treatment alone carried a significantly increased risk for IS or CVD compared to ADT-naïve patients.

Conclusions: The current study based on Asian population suggests that treatment with neither $\mathrm{GnRH}$ agonist nor antiandrogens increases the risk of cardio-cerebrovascular disease compared to patients with ADT-naïve prostate cancer.
\end{abstract}

Key words: Prostatic Neoplasms, Gonadotropin-Releasing Hormone, Antiandrogens, Cerebrovascular Disease, Cardiovascular Diseases

\section{Introduction}

Androgen deprivation therapy (ADT) is routinely used to treat patients with advanced prostate cancer (PCa)[1]. It is well known that ADT causes side effects such as hot flushes, fatigue, decreased libido, and erectile dysfunction [2]. Recent multiple observational studies have shown that men treated with $\mathrm{ADT}$ are at an increased risk of developing cardio-cerebro-vascular disease (CCVD), 
including myocardial infarction (MI) and ischemic stroke (IS) [3-6]. These studies have led to issues and advisories regarding the relevance of ADT to CCVD [7]. The assumed biological mechanism underlying these observational studies is the link between ADT and the formation of atherosclerotic plaque. Recent animal studies suggest that ADT contributes to the development of atherosclerosis [8, 9]. ADT induces metabolic changes that promote the development and progression of atherosclerotic plaques under the effect of hormonal factors on plaque growth, rupture, and thrombosis [10].

However, these theories remain inconclusive. Further studies are needed to confirm the association between ADT and CCVD. In particular, physiological, genetic, and ethnic differences might contribute to metabolic changes associated with ADT. However, most studies investigating the association between ADT and CCVD involved the Western population. Only a few studies involved Asian populations, including a small number of patients in a single institution, which does not represent the whole population.

Therefore, this population-based study used the National Health Insurance Service-Elderly Cohort Database (NHIS-ECD). To determine whether the use of ADT was associated with an increased risk of CCVD in patients with PCa.

\section{Patients and Methods}

\section{Data source}

This study used claims data acquired from NHIS-ECD released by the Korean National Health Insurance Service (KNHIS). The KNHIS provides data sharing service to support policy and academic investigations using claims data collected under the national health insurance program. The cohort members of NHIS-ECD were selected by a simple random sampling method from patients aged 60 years or older who qualified for the health insurance program in 2012. These cohort members were followed up for 12 years until 2013 unless the individual was disqualified due to death or emigration. The NHIS-ECD contains information regarding 558,147 elderly patients, accounting for approximately $10 \%$ of the entire South Korean patients aged 60 years or older. The following variables were included in the NHIS-ECD data: socio-economic status (including death and disability), principal diagnosis or comorbidities based on the International Classification of Disease, 10th Revision (ICD-10), surgical procedures, and drug prescription (generic name, dosage, and prescription duration). Because patient data in NHIS-ECD were fully de-identified, the requirement for Institutional Review Board approval was waived.

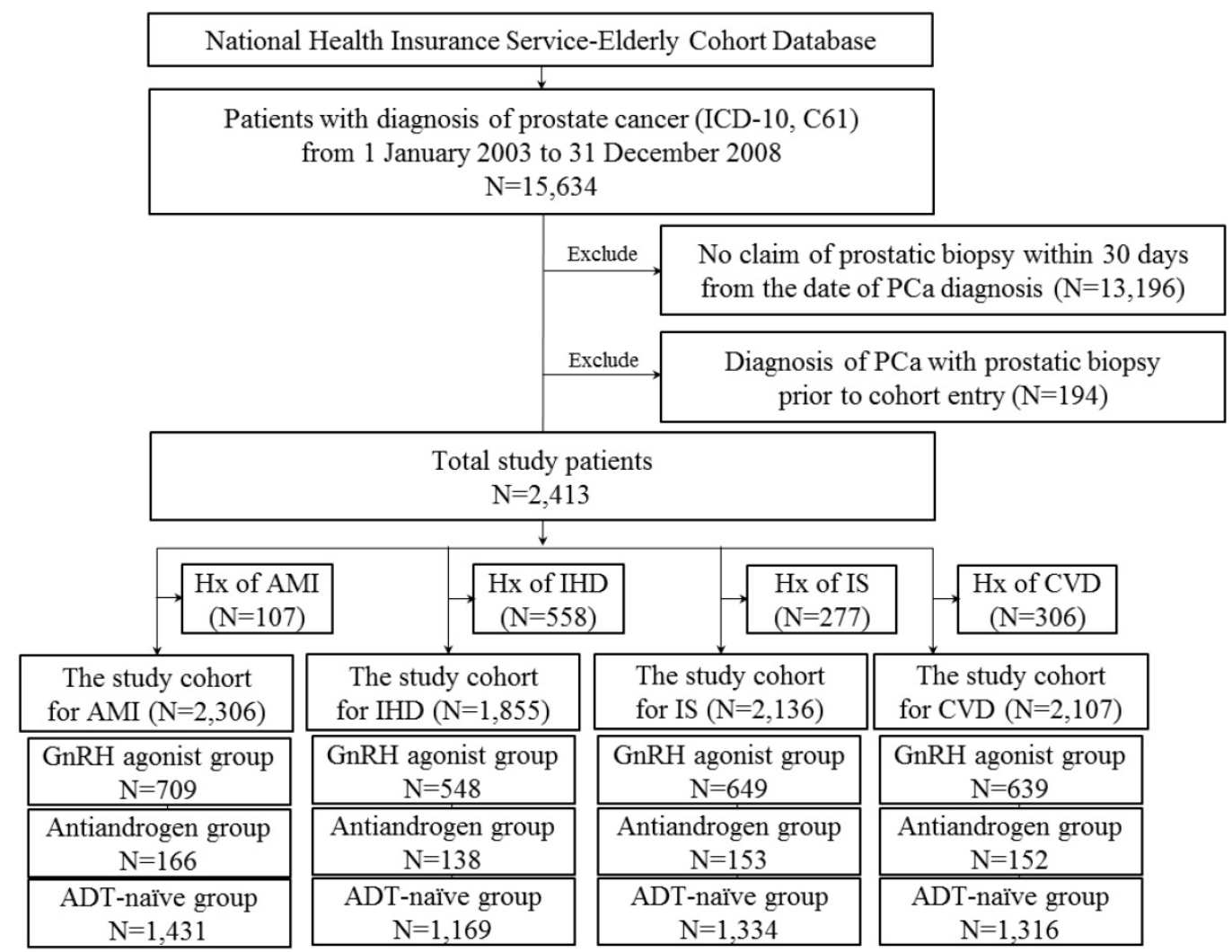

Figure 1. Flow diagram for selection of the study cohort. 


\section{Study cohort}

Male patients aged 60 years or older with a first diagnosis of PCa between January 2003 and December 2008, and prostatic biopsy within 30 days from the date of the first diagnosis were included in this study (Figure 1).

Patients with prevalent CCVD who had diagnosis of either condition prior to cohort entry or 6 months after cohort entry were excluded. Study subjects were classified according to treatment type. Patients treated with gonadotropin-releasing hormone [GnRH] agonists within 6 months of $\mathrm{PCa}$ diagnosis were defined as GnRH agonist users. Patients treated with antiandrogens alone within six months of diagnosis were defined as antiandrogen users. We included patients who were treated for at least 6 months (GnRH agonists or antiandrogens only). Patients who received neither type of treatment were defined as ADT-naïve.

\section{Outcomes and Covariates}

CCVD events included cardiovascular and cerebrovascular events. Cardiovascular events were classified into acute myocardial infarction (AMI) and ischemic heart disease (IHD) while cerebrovascular events were classified into ischemic stroke (IS) and cerebrovascular disease (CVD). Outcomes of interest included the first occurrence of AMI, IHD, IS, and CVD. Incident AMI was defined as a hospitalization or emergency room (ER) visit with an ICD-10 code of I21-I23 or I25.2 in the primary or secondary diagnosis code. Similarly, IHD, IS, or CVD was defined as a hospitalization or ER visit with an ICD-10 code of I20-I25 for IHD, I63 for IS, and I60-I69 for CVD in the primary or secondary diagnosis code.

Baseline covariates were derived from medical claims during 12 months preceding the cohort entry. The variables included age, year of diagnosis of PCa, diabetes mellitus (ICD-10 code of E10-14), hypertension (ICD-10 code of I10, I11, I13, and I14), and Charlson comorbidity index (CCI). CCI was calculated from medical claims one year before $\mathrm{PCa}$ diagnosis using the method proposed by Quan et al [11].

\section{Statistical analysis}

Each patient was followed up from the cohort entry date until the diagnosis of the first CCVD event of interest, the start of another ADT, death, or the end of study period (31 December 2013). Analysis of variance (ANOVA) was used to compare means while Chi-square test was used to compare the proportions of study patients according to the different treatment regimens. We calculated the incidence rates of AMI, IHD, IS, and CVD during treatment with GnRH agonists, antiandrogens alone, or ADT-naïve interventions. Survival analyses were performed using Cox proportional-hazards regression. All covariates in Table 1 were adjusted to calculate the hazard ratios and their $95 \%$ confidence intervals. All analyses were performed using the SAS software version 9.4 (SAS Institute, Cary, NC, USA).

\section{Results}

The current study cohort comprising a total of 2,413 prostate cancer patients included 738 who received GnRH agonist, 173 treated with antiandrogen alone, and 1502 exposed to neither type of treatment. The development of IHD, AMI, IS, and CVD in the study cohort was followed for a median of 5.79 years (range, 33 days to 11.0 years), 5.84 years (range, 33 days to 11.0 years), 5.62 years (range, 4 days to 11.0 years), and 5.50 years (range, 4 days to 11.0 years) following PCa diagnosis, respectively.

\section{Overall patient characteristics}

The overall patient characteristics in the prostate cancer study cohort are summarized in Table 1 . Significant baseline differences were found in age, CCI, and BMI between ADT cohort and ADT-naïve cohort. Patients in the ADT cohort (GnRH agonist or antiandrogen only) were more likely to be older with a higher CCI. They also showed a distinct difference in age distribution. More than $20 \%$ of the ADT cohort were men aged above 80 years. Conversely, only $7.7 \%$ of men in the ADT-naïve cohort were aged above 80 years.

\section{Analysis of cardiovascular event outcome}

Overall, the 5-year AMI-free and IHD-free rates were $97.8 \%$ and $94.0 \%$, respectively, in the current cohort. The AMI-free rates of patients treated with GnRH agonists, antiandrogens, and those who were ADT-naïve were $97.0 \%, 96.5 \%$, and $98.3 \%$, respectively, while the IHD-free rates were 93.2\%, $92.3 \%$, and $94.5 \%$, respectively (Figure 2). Results of Cox proportional-hazards analysis stratified into AMI and IHD in the current cohort are shown in Table 2.

In crude Cox proportional-hazards models, the use of GnRH agonist in patients resulted in a significantly increased risk of AMI (hazard ratio/HR: 1.996, $P=0.01$ ) compared to ADT-naïve patients (Table 2) as shown by the Kaplan-Meier survival curve $(2 \mathrm{~A}$; log-rank $P$-value $=0.04)$. Conversely, treatment with antiandrogen alone was not associated with a significantly increased risk of AMI (HR: 1.799; $P=0.27)$. In multivariate Cox proportional-hazards models, the use of GnRH agonists (HR: 1.503; $P=0.17$ ) or antiandrogen only (HR: $1.208 ; \mathrm{P}=0.73$ ) in PCa patients did not significantly increase the risk of AMI 
after adjusting for other covariates compared to ADT-naïve patients.

Concerning IHD, the Cox proportional hazards models showed that PCa patients who received GnRH agonist did not have a significantly increased risk compared to ADT-naïve patients in crude analysis (HR: $1.257 ; \mathrm{P}=0.24$ ) or multivariate analysis (HR 1.107; $\mathrm{P}=0.62$ ). The use of antiandrogen alone did not show a significantly increased risk of IHD compared to ADT-naïve patients in crude analysis (HR: 1.540; $\mathrm{P}=0.22$ ) or multivariate analysis (HR: 1.337; $\mathrm{P}=0.42$ ). These results were consistent with Kaplan-Meier survival curves, showing no significant difference in the survival of patients exposed to GnRH agonist or antiandrogen alone compared to ADT-naïve group in each event category $(\mathrm{P}=0.29$, log-rank test for IHD).

\section{Analysis of cerebrovascular event outcome}

Overall, the 5-year IS-free and CVD-free rates were $95.3 \%$ and $94.1 \%$, respectively. The IS-free rates for $\mathrm{PCa}$ patients exposed to GnRH agonist, antiandrogens, and those who were ADT-naïve were $94.8 \%, 94.7 \%$, and $95.5 \%$, respectively, while the CVD-free rates were $92.9 \%, 93.3 \%$, and $94.6 \%$, respectively (Figure 3 ).

Table 1. Overall patient characteristics of the cohorts

\begin{tabular}{|c|c|c|c|c|c|c|c|c|}
\hline & & \multicolumn{2}{|c|}{$\begin{array}{l}\text { ADT-naïve } \\
(\mathrm{N}=1502)\end{array}$} & \multicolumn{2}{|c|}{$\begin{array}{l}\text { GnRH agonists } \\
(\mathrm{N}=738)\end{array}$} & \multicolumn{2}{|c|}{$\begin{array}{l}\text { Antiandrogen only } \\
(\mathrm{N}=173)\end{array}$} & \multirow[t]{2}{*}{ p-value } \\
\hline & & $\mathrm{N}$ & $\%$ & $\mathrm{~N}$ & $\%$ & $\mathrm{~N}$ & $\%$ & \\
\hline Age & Mean, SE & 71.1 & 5.5 & 74.2 & 6.1 & 74.1 & 6.7 & $<0.01$ \\
\hline \multirow[t]{3}{*}{ Age group } & $60-69$ & 655 & 43.6 & 190 & 25.7 & 49 & 28.3 & \\
\hline & $70-79$ & 731 & 48.7 & 393 & 53.3 & 83 & 48.0 & \\
\hline & $80<$ & 116 & 7.7 & 155 & 21.0 & 41 & 23.7 & \\
\hline \multirow{6}{*}{$\begin{array}{l}\text { Year of diagnosis of } \\
\text { prostate cancer }\end{array}$} & 2003 & 205 & 13.6 & 71 & 9.6 & 35 & 20.2 & $<0.01$ \\
\hline & 2004 & 245 & 16.3 & 89 & 12.1 & 28 & 16.2 & \\
\hline & 2005 & 208 & 13.8 & 108 & 14.6 & 24 & 13.9 & \\
\hline & 2006 & 247 & 16.4 & 119 & 16.1 & 32 & 18.5 & \\
\hline & 2007 & 280 & 18.6 & 162 & 22.0 & 25 & 14.5 & \\
\hline & 2008 & 317 & 21.1 & 189 & 25.6 & 29 & 16.8 & \\
\hline \multirow[t]{2}{*}{ Comorbidity } & Diabetes & 432 & 28.8 & 227 & 30.8 & 51 & 29.5 & 0.62 \\
\hline & Hypertension & 817 & 54.4 & 430 & 58.3 & 90 & 52.0 & 0.14 \\
\hline \multirow[t]{5}{*}{ CCI } & Mean, SE & 4.2 & 2.3 & 4.9 & 3.0 & 4.8 & 2.9 & $<0.01$ \\
\hline & 2 & 339 & 22.6 & 144 & 19.5 & 38 & 22.0 & \\
\hline & 3 & 339 & 22.6 & 155 & 21.0 & 35 & 20.2 & \\
\hline & 4 & 294 & 19.6 & 135 & 18.3 & 36 & 20.8 & \\
\hline & $5<$ & 470 & 31.3 & 304 & 41.2 & 64 & 37.0 & \\
\hline \multirow[t]{16}{*}{ Comorbidities in CCI } & Myocardial infarction & 40 & 2.7 & 18 & 2.4 & 6 & 3.5 & 0.75 \\
\hline & Congestive heart failure & 75 & 5.0 & 57 & 7.7 & 15 & 8.7 & 0.01 \\
\hline & Peripheral vascular disease & 145 & 9.7 & 102 & 13.8 & 11 & 6.4 & $<0.01$ \\
\hline & Cerebrovascular disease & 228 & 15.2 & 142 & 19.2 & 28 & 16.2 & 0.05 \\
\hline & Dementia & 20 & 1.3 & 18 & 2.4 & 3 & 1.7 & 0.16 \\
\hline & Chronic pulmonary disease & 557 & 37.1 & 254 & 34.4 & 72 & 41.6 & 0.17 \\
\hline & Rheumatic disease & 74 & 4.9 & 38 & 5.1 & 6 & 3.5 & 0.65 \\
\hline & Peptic ulcer disease & 523 & 34.8 & 273 & 37.0 & 44 & 25.4 & 0.02 \\
\hline & Mild liver disease & 331 & 22.0 & 177 & 24.0 & 36 & 20.8 & 0.5 \\
\hline & Diabetes without chronic complication & 404 & 26.9 & 208 & 28.2 & 47 & 27.2 & 0.81 \\
\hline & Diabetes with chronic complication & 136 & 9.1 & 76 & 10.3 & 13 & 7.5 & 0.44 \\
\hline & Hemiplegia or paraplegia & 22 & 1.5 & 19 & 2.6 & 4 & 2.3 & 0.17 \\
\hline & Renal disease & 35 & 2.3 & 23 & 3.1 & 5 & 2.9 & 0.53 \\
\hline & Moderate or severe liver disease & 13 & 0.9 & 7 & 0.9 & 2 & 1.2 & 0.92 \\
\hline & Metastatic solid tumor & 81 & 5.4 & 107 & 14.5 & 29 & 16.8 & $<0.01$ \\
\hline & AIDS/HIV & 0 & 0.0 & 0 & 0.0 & 0 & 0.0 & - \\
\hline
\end{tabular}

Table 2. Crude- and multivariable-adjusted hazard ratios for cardiovascular disease according to treatment modality in prostate cancer cohort

\begin{tabular}{|c|c|c|c|c|c|c|c|c|c|}
\hline & \multirow[t]{2}{*}{ Treatment group } & \multirow[t]{2}{*}{$\mathbf{N}$} & \multirow[t]{2}{*}{ Event } & \multirow[t]{2}{*}{ Person year } & \multirow[t]{2}{*}{ Incidence } & \multicolumn{4}{|c|}{ H.R (95\% Confidence interval) } \\
\hline & & & & & & Crude & & Adju & \\
\hline \multirow[t]{3}{*}{ IHD } & No medication & 1169 & 84 & 7128.83 & 11.78 & 1 & Ref. & & Ref. \\
\hline & GnRH agonists & 548 & 39 & 2723.23 & 14.32 & 1.257 & $(0.858-1.840)$ & 1.107 & $(0.743-1.651)$ \\
\hline & Antiandrogen only & 138 & 9 & 536.26 & 16.78 & 1.54 & $(0.774-3.067)$ & 1.337 & $(0.661-2.706)$ \\
\hline \multirow[t]{3}{*}{ AMI } & No medication & 1431 & 29 & 8865.31 & 3.27 & 1 & Ref. & & Ref. \\
\hline & GnRH agonists & 709 & 23 & 3549.95 & 6.48 & 1.996 & $(1.151-3.461)$ & 1.503 & $(0.841-2.688)$ \\
\hline & Antiandrogen only & 166 & 4 & 681.74 & 5.87 & 1.799 & $(0.631-5.127)$ & 1.208 & $(0.413-3.529)$ \\
\hline
\end{tabular}

* Adjusted for all covariated in Table 1 
A

AMI

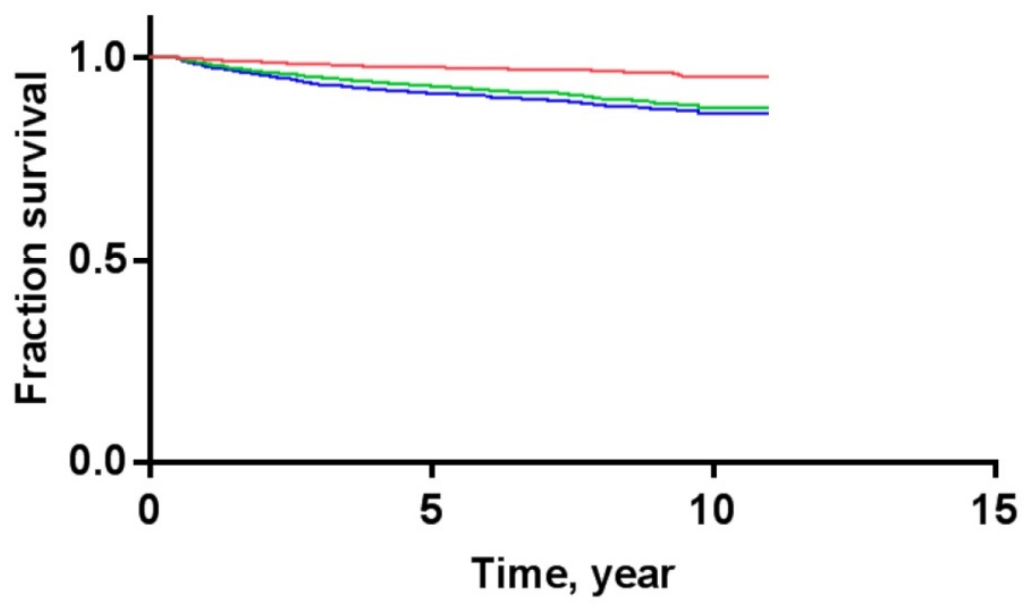

- No medication - $\begin{gathered}\text { Antiandrogen only } \\ \text { IHD }\end{gathered}-\mathrm{GnRH}$ agonist

B

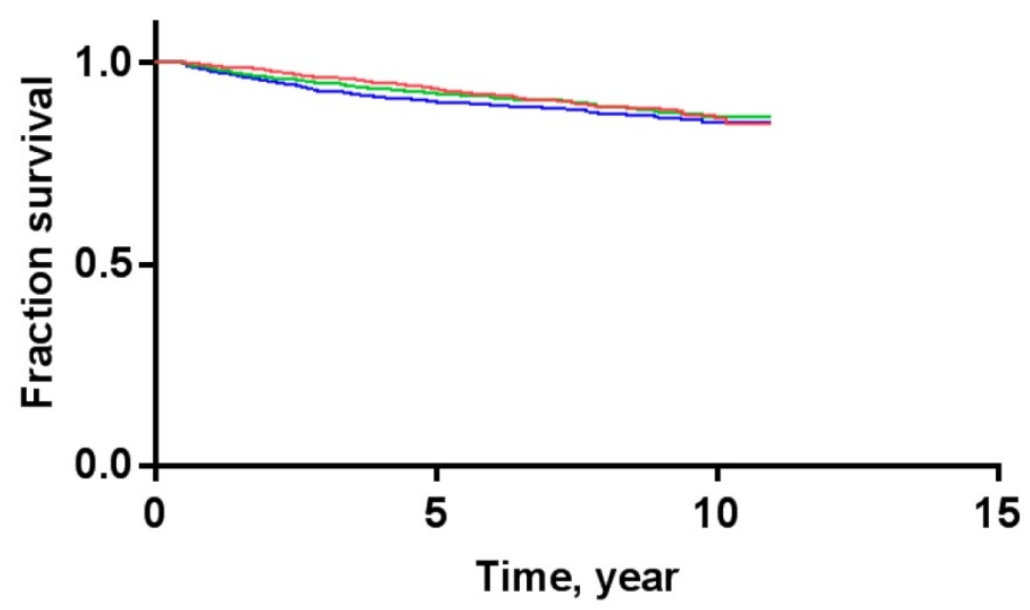

No medication - Antiandrogen only

- GnRH agonist

Figure 2. Kaplan-Meier survival curves for patients with AMI (A) and IHD (B) according to prostate cancer treatment modality.

The results of Cox proportional-hazards analysis stratified into IS and CVD in the current cohort are shown in Table 3. The Cox proportional-hazards models showed that PCa patients who were treated with GnRH agonist did not carry a significantly increased risk of IS or CVD compared to ADT-naïve patients in crude analysis (for IS: HR, 1.156; $\mathrm{P}=$ 0.4633; for CVD: HR, 1.111; $\mathrm{P}=0.5579$ ) or multivariate analysis (for IS: HR, 0.978; P = 0.9128; for CVD: HR, 0.939; $\mathrm{P}=0.7339)$. $\mathrm{PCa}$ patients who used antiandrogens alone did not show a significantly increased risk of IS or CVD either compared to ADT-naïve patients in crude analysis (for IS: HR, 1.001; $\mathrm{P}=0.9981$; for CVD: HR, 0.926; $\mathrm{P}=0.8435$ ) or multivariate analysis (for IS: $\mathrm{HR}, 0.822 ; \mathrm{P}=0.6484$; for CVD: HR, 0.760; $\mathrm{P}=0.4891)$. These results were consistent with Kaplan-Meier survival curves (Figure 3), showing no significant difference in survival of patients exposed to $\mathrm{GnRH}$ agonist or antiandrogen only compared to ADT-naïve patients in each event category (for IS: log-rank test $P$-value $=0.76$; for CVD: log-rank test $P$-value $=0.81$ ).

\section{Discussion}

The key findings of this Asian population-based study are as follows. First, prostate cancer patients who received GnRH agonist or antiandrogens only did not show an increased risk of cardiovascular disease compared to ADT-naïve patients. Second, the use of GnRH agonist or antiandrogens was not a significant risk factor for developing cerebrovascular disease in Asian men with prostate cancer. 
Table 3. Crude- and multivariable-adjusted hazard ratios for cerebrovascular disease according to treatment modality in prostate cancer cohort

\begin{tabular}{|c|c|c|c|c|c|c|c|c|c|}
\hline & \multirow[t]{2}{*}{ Treatment group } & \multirow[t]{2}{*}{$\mathrm{N}$} & \multirow[t]{2}{*}{ Event } & \multirow[t]{2}{*}{ Person year } & \multirow[t]{2}{*}{ Incidence } & \multicolumn{4}{|c|}{ H.R (95\% Confidence interval) } \\
\hline & & & & & & Crude & & Adjus & \\
\hline \multirow[t]{3}{*}{ IS } & No medication & 1334 & 86 & 8185.07 & 10.51 & 1 & Ref. & & Ref. \\
\hline & GnRH agonists & 649 & 37 & 3200.95 & 11.56 & 1.156 & $(0.785-1.702)$ & 0.978 & $(0.655-1.460)$ \\
\hline & Antiandrogen only & 153 & 6 & 609.68 & 9.84 & 1.001 & $(0.437-2.292)$ & 0.822 & $(0.354-1.910)$ \\
\hline \multirow[t]{3}{*}{ CVD } & No medication & 1316 & 107 & 8039.8 & 13.31 & 1 & Ref. & & Ref. \\
\hline & GnRH agonists & 639 & 44 & 3132.05 & 14.05 & 1.111 & $(0.781-1.581)$ & 0.939 & $(0.652-1.352)$ \\
\hline & Antiandrogen only & 152 & 7 & 609.07 & 11.49 & 0.926 & $(0.431-1.990)$ & 0.76 & $(0.349-1.655)$ \\
\hline
\end{tabular}

* Adjusted for all covariated in Table 1

A

IS

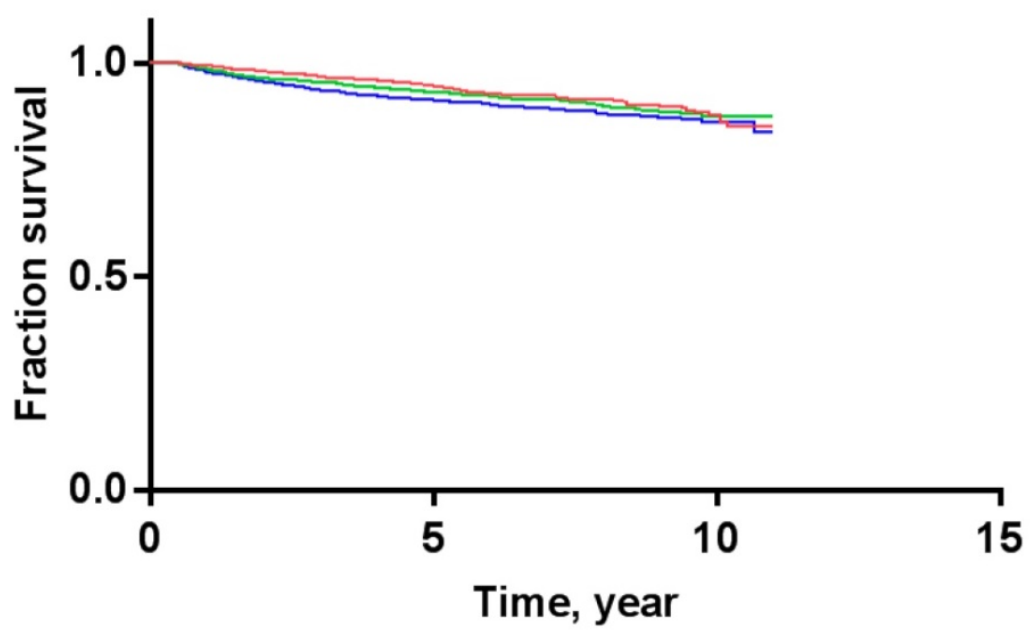

\section{No medication \\ - Antiandrogen only CVD}

B

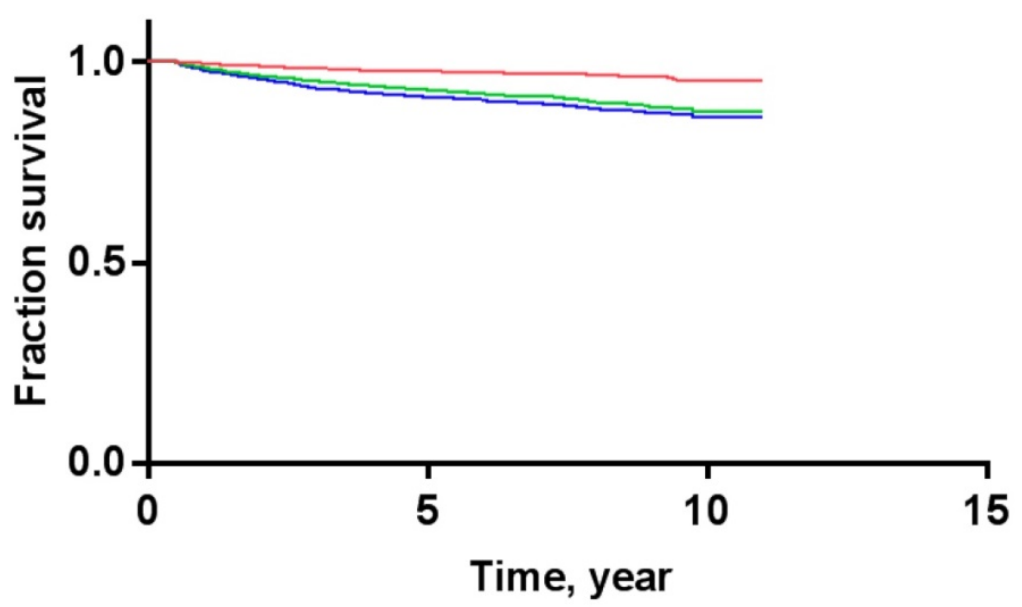

No medication

Antiandrogen only

GnRH agonist

Figure 3. Kaplan-Meier survival curves for patients with IS (A) and CVD (B) according to prostate cancer treatment modality.

Despite ongoing review, the FDA drug safety communication has warned that ADT increases the risk of certain cardiovascular diseases, and physicians should monitor their development [12]. These recommendations are consistent with the Science
Advisory issued by the American Heart Association, American Cancer Society, and American Urological Association. Recent meta-analyses using observational data of ADT and the risk of CCVD events in men with prostate cancer have also suggested that 
ADT might increase the risk of CCVD $[13,14]$. The pathogenesis of ADT-induced CCVD may involve changes in body composition and metabolism. ADT has been shown to suppress testosterone production, which leads to decreased lean muscle mass but increased fat mass [15]. Another suggested mechanism is that the low level of testosterone induces insulin resistance and the adverse lipid profile, which increase vessel wall thickness and trigger atherosclerosis, resulting in endothelial dysfunction [16, 17].

However, the relationship between ADT and CCVD remains controversial. In addition, most studies investigating the effect of ADT on CCVD have been based on western populations including Caucasians. As mentioned above, the pathophysiological events underlying changes in body composition, especially fat mass, play a key role in the development of CCVD. Significant differences in body composition and fat mass have been found between western population and Asians. In general, the prevalence of obesity is significantly higher in western population than in Asian population [18]. In the United States, 33\% of the population is obese. In the UK, $26.9 \%$ of the residents are obese. In Korea and Japan, the percentage of obese people is relatively very low [19]. A small amount of fat mass in the Asian population has an impact on the risk of CCVD following ADT.

Physiological and genetic differences according to ethnicity also affect the relationship between ADT and CCVD. Differences in androgen receptor (AR) CAG repeat length polymorphism [20-22] and deletion of UGT2B genes $[23,24]$ contribute to differences in the development of CCVD between Asians and Caucasians. These ethnic differences affect the development of CCVD following ADT. Therefore, further investigations are needed to determine the association between ADT and CCVD in Asian population.

Our results are in agreement with previous Asian population-based studies, including the Japanese population [25] and the Taiwanese population [26]. The Japanese population study showed no increased risk of coronary heart disease in patients exposed to ADT. The Taiwanese population study showed no increased risk of IS following ADT [27]. However, other Asian population studies suggested increased risks of CCVD in patients receiving ADT [28-30]. Although such discrepancies cannot be explained, studies reporting a positive association between ADT and CCVD involve relatively small numbers of subjects at a single institution. Therefore, they cannot accurately represent the population. The current study is a nationwide representative analysis of Korean population. This study analyzed data from NHIS-ECD, which sampled more than 55 million people aged 60 years or older through national health insurance service registration. The study represents approximately $10 \%$ of all South Korean patients aged 60 years or older. It therefore provides a highly representative cohort of the Korean population.

It is worth noting that this is the first population-based observational study to investigate the relationship between ADT and the risk of CCVD among Koreans, a population with homogeneous ethnicity. To the best of our knowledge, the sample size and observation period in this study are larger than those of previous studies involving Asian population. In addition, we carefully defined the potential confounders at baseline. Male patients with a history of any cardiovascular disease (except hypertension, ischemic, or hemorrhagic stroke) and renal failure were excluded from the analysis, which was thus more reliable by controlling potential confounders. In addition, those who were aged above 40 years in South Korea were advised to undergo health examination including electrocardiography and lipid profile. Any abnormal findings were covered by NHIS for further medical evaluation. Therefore, the analysis entailing frequent and widespread medical evaluation has considerable strength and power.

Our study has some limitations due to potential confounding factors associated with lifestyle. Smoking status and obesity are known risk factors for CCVD. However, they were not evaluated in the current study. Another limitation was that patients were not categorized by cancer status such as stage, metastasis, and Gleason score in the current study because cancer profile was unavailable in the current database. However, the extent or staging of prostate cancer might not modify CCVD risk. Lastly, the potential for disease misclassification based on claim database was another limitation. The Korean NHISS covers almost all medical costs of patients with major diseases such as malignancy, coronary heart disease, and stroke. Patients only need to pay $5 \%$ of the total medical cost. However, the registration and certification criteria for patients with major diseases are stringent, which ensures sufficient reliability and trustworthiness of medical claims.

\section{Conclusions}

The current study based on nation-wide population in Korea suggests that the use of ADT in prostate cancer patients does not increase the risk of CCVD compared to ADT-naïve patients. Although it is not sufficient to establish the association between 
ADT and CCVD, the Asian population differs from western population. Therefore, further well-designed studies including different regions and populations are needed to elucidate the increased risk of CCVD following ADT.

\section{Acknowledgments}

\section{Funding}

This work was supported by the National Research Foundation of Korea (NRF) grant funded by the Korea government (No. NRF- 2018R1D1A1B0 7049542).

\section{Abbreviations}

ADT: Androgen deprivation therapy; PCa: prostate cancer; CCVD: cardio-cerebro vascular disease; MI: myocardial infarction; IS: ischemic stroke; NHIS-ECD: National Health Insurance ServiceElderly Cohort Database; KNHIS: Korean National Health Insurance Service; ICD-10: the International Classification of Disease, 10th Revision; GnRH: gonadotropin releasing hormone; AMI: acute myocardial infarction; IHD: ischemic heart disease; IS: ischemic stroke; CVD: cerebrovascular disease; ER: emergency room; CCI: Charlson comorbidity index; ANOVA: Analysis of variance; HR: hazard ratio.

\section{Competing Interests}

The authors have declared that no competing interest exists.

\section{References}

1. Bekelman JE, Mitra N, Handorf EA, et al. Effectiveness of androgen-deprivation therapy and radiotherapy for older men with locally advanced prostate cancer. J Clin Oncol. 2015; 33: 716-22.

2. Sharifi N, Gulley JL, Dahut WL. Androgen deprivation therapy for prostate cancer. JAMA. 2005; 294: 238-44.

3. Zhao J, Zhu S, Sun L, et al. Androgen deprivation therapy for prostate cancer is associated with cardiovascular morbidity and mortality: a meta-analysis of population-based observational studies. PLoS One. 2014; 9: e107516.

4. Bosco C, Crawley D, Adolfsson J, Rudman S, Van Hemelrijck M. Quantifying the evidence for the risk of metabolic syndrome and its components following androgen deprivation therapy for prostate cancer: a meta-analysis. PLoS One. 2015; 10: e0117344.

5. Conteduca V, Di Lorenzo G, Tartarone A, Aieta M. The cardiovascular risk of gonadotropin releasing hormone agonists in men with prostate cancer: an unresolved controversy. Crit Rev Oncol Hematol. 2013; 86: 42-51.

6. Albertsen PC, Klotz L, Tombal B, Grady J, Olesen TK, Nilsson J. Cardiovascular morbidity associated with gonadotropin releasing hormone agonists and an antagonist. Eur Urol. 2014; 65: 565-73.

7. Levine GN, D'Amico AV, Berger P, et al. Androgen-deprivation therapy in prostate cancer and cardiovascular risk: a science advisory from the American Heart Association, American Cancer Society, and American Urological Association: endorsed by the American Society for Radiation Oncology. Circulation. 2010; 121: 833-40.

8. Nettleship JE, Jones TH, Channer KS, Jones RD. Physiological testosterone replacement therapy attenuates fatty streak formation and improves high-density lipoprotein cholesterol in the Tfm mouse: an effect that is independent of the classic androgen receptor. Circulation. 2007; 116: 2427-34.

9. Wu J, Hadoke PW, Mair I, et al. Modulation of neointimal lesion formation by endogenous androgens is independent of vascular androgen receptor. Cardiovasc Res. 2014; 103: 281-90.

10. Zareba P, Duivenvoorden W, Leong DP, Pinthus JH. Androgen deprivation therapy and cardiovascular disease: what is the linking mechanism? Ther Adv Urol. 2016; 8: 118-29.
11. Quan H, Sundararajan V, Halfon $P$, et al. Coding algorithms for defining comorbidities in ICD-9-CM and ICD-10 administrative data. Med Care. 2005; 43: 1130-9.

12. [Internet] FDA. FDA Drug Safety Communication: Update to Ongoing Safety Review of GnRH Agonists and Notification to Manufacturers of GnRH Agonists to Add New Safety Information to Labeling Regarding Increased Risk of Diabetes and Certain Cardiovascular Diseases. https://www.fda.gov/Drugs/DrugSafety/ucm229986.htm

13. Bosco C, Bosnyak Z, Malmberg A, Adolfsson J, Keating NL, Van Hemelrijck M. Quantifying observational evidence for risk of fatal and nonfatal cardiovascular disease following androgen deprivation therapy for prostate cancer: a meta-analysis. Eur Urol. 2015; 68: 386-96.

14. Meng F, Zhu S, Zhao J, et al. Stroke related to androgen deprivation therapy for prostate cancer: a meta-analysis and systematic review. BMC Cancer. 2016; 16: 180.

15. Saylor PJ, Smith MR. Metabolic complications of androgen deprivation therapy for prostate cancer. J Urol. 2013; 189: S34-44.

16. Traish AM, Saad F, Feeley RJ, Guay A. The dark side of testosterone deficiency: III. Cardiovascular disease. J Androl. 2009; 30: 477-94.

17. Traish AM, Saad F, Guay A. The dark side of testosterone deficiency: II. Type 2 diabetes and insulin resistance. J Androl. 2009; 30: 23-32.

18. [Internet] OECD. Obesity Update. http://www.oecd.org/health/obesityupdate.htm

19. [Internet] Smith O. World Obesity Day: Which countries have the biggest weight problem? http://www.telegraph.co.uk/travel/maps-and-graphics/the-most-obese-fatt est-countries-in-the-world/

20. Li J, Mercer E, Gou X, Lu YJ. Ethnical disparities of prostate cancer predisposition: genetic polymorphisms in androgen-related genes. Am J Cancer Res. 2013; 3: 127-51.

21. Hsing AW, Gao YT, Wu G, et al. Polymorphic CAG and GGN repeat lengths in the androgen receptor gene and prostate cancer risk: a population-based case-control study in China. Cancer Res. 2000; 60: 5111-6.

22. Möhlig M, Arafat AM, Osterhoff MA, et al. Androgen receptor CAG repeat length polymorphism modifies the impact of testosterone on insulin sensitivity in men. Eur J Endocrinol. 2011; 164: 1013-8.

23. Nadeau G, Bellemare J, Audet-Walsh É, et al. Deletions of the androgen-metabolizing UGT2B genes have an effect on circulating steroid levels and biochemical recurrence after radical prostatectomy in localized prostate cancer. J Clin Endocrinol Metab. 2011; 96: E1550-7.

24. Guillemette C, Lévesque E, Harvey M, Bellemare J, Menard V. UGT genomic diversity: beyond gene duplication. Drug Metab Rev. 2010; 42: 24-44.

25. Akaza H. Future prospects for luteinizing hormone-releasing hormone analogues in prostate cancer treatment. Pharmacology. 2010; 85: 110-20.

26. Wang LH, Liu CK, Chen $\mathrm{CH}$, Kao LT, Lin HC, Huang CY. No increased risk of coronary heart disease for patients receiving androgen deprivation therapy for prostate cancer in Chinese/Taiwanese men. Andrology. 2016; 4: 128-32.

27. Chung SD, Chen YK, Wu FJ, Lin HC. Hormone therapy for prostate cancer and the risk of stroke: a 5-year follow-up study. BJU Int. 2012; 109: 1001-5.

28. Teoh JY, Chan SY, Chiu PK, et al. Risk of acute myocardial infarction after androgen-deprivation therapy for prostate cancer in a Chinese population. BJU Int. 2015; 116: 382-7.

29. Teoh JY, Chiu PK, Chan SY, et al. Risk of ischemic stroke after androgen deprivation therapy for prostate cancer in the Chinese population living in Hong Kong. Jpn J Clin Oncol. 2015; 45: 483-7.

30. Huang G, Yeung CY, Lee KK, et al. Androgen deprivation therapy and cardiovascular risk in chinese patients with nonmetastatic carcinoma of prostate. J Oncol. 2014; 2014: 529468. 